\title{
Non-noxious skin stimulation activates the nucleus basalis of Meynert and promotes NGF secretion in the parietal cortex via nicotinic $\mathrm{ACh}$ receptors
}

\author{
Harumi Hotta $\cdot$ Nobuhiro Watanabe $\cdot$ \\ Mathieu Piché • Sanae Hara • Takashi Yokawa • \\ Sae Uchida
}

Received: 27 February 2014/ Accepted: 20 March 2014/Published online: 7 May 2014

(c) The Author(s) 2014. This article is published with open access at Springerlink.com

\begin{abstract}
The effects of non-noxious skin stimulation on nerve growth factor (NGF) secretion in the parietal cortex were examined in anesthetized rats. Innocuous skin stimulation was delivered to the left hindlimb with a soft-hair brush. Extracellular NGF in the right parietal cortex was collected by microdialysis methods using a protein-permeable probe and was measured using an enzyme-linked immune-sorbent assay. Brushing produced a significant increase in extracellular NGF levels. This NGF response was not observed in rats pretreated with a nicotinic ACh receptor (nAChR) antagonist mecamylamine. We further examined whether brushing could activate the basal forebrain nucleus (nucleus basalis of Meynert, NBM), which is the main source of cholinergic fibers in the cerebral cortex, by means of functional MRI. The blood oxygen leveldependent signal in the right NBM was significantly higher during brushing compared to baseline. The results suggest that non-noxious skin stimulation activates NBM and promotes NGF secretion in the parietal cortex via nAChRs.
\end{abstract}

Keywords Physical stimulation - Nerve growth factor Nicotinic receptor - Functional magnetic resonance imaging · Nucleus basalis of Meynert

H. Hotta $(\bowtie) \cdot$ N. Watanabe $\cdot$ S. Hara $\cdot$ S. Uchida

Department of Autonomic Neuroscience, Tokyo Metropolitan

Institute of Gerontology, 35-2 Sakaecho, Itabashi-ku,

Tokyo 173-0015, Japan

e-mail: hhotta@tmig.or.jp

\section{Piché}

Department of Chiropractic,

Université du Québec à Trois-Rivières, Quebec, Canada

T. Yokawa

BioView, Tokyo, Japan

\author{
Abbreviations \\ BOLD Blood oxygen level-dependent \\ ELISA Enzyme-linked immune-sorbent assay \\ fMRI Functional magnetic resonance imaging \\ nAChRs Nicotinic ACh receptors \\ NBM The nucleus basalis of Meynert \\ NGF Nerve growth factor \\ ROI Region of interest
}

\section{Introduction}

Non-noxious mechanical skin stimulation produces not only somatosensory sensation but also has various effects on visceral functions independent of sensation in anesthetized animals and conscious humans [1-7]. Additionally, non-noxious skin stimulation may improve cognitive dysfunctions in patients with dementia [8-10], and motor functions in patients and animal models of cerebral infarction [11-15]. However, the mechanisms of these effects on the brain have not been clarified.

Nerve growth factor (NGF) is a neurotrophic factor that is produced and secreted by cerebral cortical neurons. Secreted NGF supports the survival and function of cholinergic basal forebrain neurons and cortical neurons essential for cognitive functions [16-19]. On the other hand, NGF secretion by cortical neurons is promoted by cholinergic inputs from the nucleus basalis of Meynert (NBM) [20]. Because of this important reciprocal influence between NGF secretion by cortical neurons and NBM cholinergic neurons, it is critical to determine what physiological stimulation promotes NGF secretion in the brain. However, there are only a few studies investigating NGF 
secretion in vivo due to the technical challenges of these methods. Therefore, in this study, we examined whether non-noxious mechanical cutaneous stimulation promotes NGF secretion in the parietal cortex in anesthetized rats using an in vivo microdialysis method with a protein permeable probe [21]. We hypothesized that non-noxious skin stimulation promotes NGF secretion in the cerebral cortex via the activation of the NBM.

We have shown that NGF secretion by cortical neurons is regulated by cholinergic inputs to the neocortex via nicotinic acetylcholine receptors (nAChRs) [20]. In anesthetized rats receiving unilateral electrical stimulation of the NBM for 100 min, NGF levels measured by enzymelinked immune-sorbent assay (ELISA) in samples from a microdialysis probe in the parietal cortex increased ipsilaterally (up to $68 \%$ ) over prestimulus levels at 200-500 min after the stimulation ended. The NGF response was abolished by the nicotinic antagonist mecamylamine, but was unaffected by the muscarinic antagonist atropine [20]. Therefore, our second aim was to examine whether the effect of skin stimulation on NGF is produced by the activation of nAChRs.

Recently, many studies have examined spatial and temporal changes in neuronal activity in various brain regions using non-invasive brain imaging techniques such as functional magnetic resonance imaging (fMRI). Measurement of blood oxygen level-dependent (BOLD) signals by fMRI showed that the parietal cortex is activated by innocuous somatic afferent stimuli in humans and animals [22-27]. However, activation of the NBM by somatosensory stimulation has not been demonstrated by brain imaging. Therefore, we thirdly examined the effect of nonnoxious skin stimulation on BOLD signal in the NBM and the parietal cortex using fMRI. Parts of the present results have been published in abstract form [28].

\section{Materials and methods}

The experiments were performed on 18 male adult Wistar rats (3-6 months old, 300-410 g). All animal experiments were conducted with the approval of and in accordance with the Guidelines for Animal Experimentation prepared by the Animal Care and Use Committee of Tokyo Metropolitan Institute of Gerontology. Of the 18 rats, 13 were used for studies on NGF secretion and 5 were used for studies of fMRI. After the end of each experiment, rats were killed by injecting an overdose of pentobarbital.

Animals were anesthetized either with halothane $(n=12)$ or urethane $(n=6)$ as described in our previous studies [21, 29]. The trachea of each rat was cannulated and respiration was maintained by a mechanical ventilator. Rectal body temperature was maintained around $37.5{ }^{\circ} \mathrm{C}$.
Halothane anesthesia (1.5\% during the surgery, and fixed at $1.0 \%$ after surgery) was used for most of the NGF experiment because this experiment requires a constant level of anesthesia for a long period of time. Urethane anesthesia (initially $1.1 \mathrm{~g} / \mathrm{kg}$, i.p.) was used for all the fMRI experiments and 1 of the NGF experiments. Additional doses of urethane were administered $(0.2 \mathrm{~g} / \mathrm{kg}$, i.p.) to maintain an anesthetic level that included the absence of spontaneous motion, corneal and withdrawal reflexes, and spontaneous ventilation.

\section{Collection and measurement of extracellular NGF}

All experimental conditions and techniques for collecting and measuring extracellular NGF in the parietal cortex using microdialysis and ELISA were similar to those described by Hotta et al. [21]. The animals were mounted in a stereotaxic instrument, and the right parietal bone and dura mater in an area of $3 \mathrm{~mm}$ in diameter at $3.5 \mathrm{~mm}$ lateral from the bregma was opened. A microdialysis probe covered with a protein permeable polysulfone membrane (NDP-I-4-03; EICOM, Kyoto, Japan) was inserted into the right parietal cortex with a lateral inclination of $30^{\circ}$ to a depth of $3 \mathrm{~mm}$ from the cortical surface at $3.5 \mathrm{~mm}$ lateral from the bregma, and was perfused at a flow rate of $2 \mu \mathrm{l} /$ min with $0.01 \mathrm{M}$ phosphate buffered saline (PBS) using a pump (ESP-64; EICOM). The outlet of the probe was connected to a silicon tube, the tip of which was set at approximately $20 \mathrm{~mm}$ below the level of the cortical surface to help the outflow of the cortical perfusate by negative hydrostatic pressure. Cortical perfusate during a period of $40 \mathrm{~min}$ from the initiation of the perfusion, while the microdialysis probe and perfusion was stabilizing, was discarded, and we began collecting cortical perfusate every $100 \mathrm{~min}$ for $600 \mathrm{~min}$. Each sample was stored at $-20{ }^{\circ} \mathrm{C}$ until assayed. The NGF concentration was measured using an ELISA kit (Promega, USA) reported to detect mature NGF, but not pro-NGF [30]. We used samples twice-diluted with the sample buffer for the assay of NGF in cortical perfusate, and calculated the original concentration of NGF.

We have previously shown that NGF levels were stable throughout this experimental period in control condition without any stimuli $[20,21]$.

\section{MRI data acquisition and analysis}

Animals were placed in a prone position and fixed into a handmade acrylic chamber. The animals' head, chest, and legs were immobilized in the chamber, which was placed in the scanner. Images were acquired using a Varian volume coil (16 cm in diameter) with a handmade circular shaped receiver surface coil $(2 \mathrm{~cm}$ in diameter) and a $4.7 \mathrm{~T}$ horizontal scanner interfaced with a Varian console (Unity 
INOVA 4.7 T). Functional MR data (BOLD response) were acquired using a single-shot gradient echo-planar imaging sequence [flip angle $(\mathrm{FA})=13^{\circ}$; repetition time $(\mathrm{TR})=5.008 \mathrm{~s}$; echo time $(\mathrm{TE})=4 \mathrm{~ms}$; field of view $(\mathrm{FOV})=3 \times 3 \mathrm{~cm}$; matrix $=32 \times 32$; number of slices $=5$; slice thickness $=0.7 \mathrm{~mm}$ ]. The 5 contiguous axial slices were collected in an interleaved manner and covered the hindpaw representation in the primary somatosensory cortex (from $2.75 \mathrm{~mm}$ posterior to $0.75 \mathrm{~mm}$ anterior to bregma). To examine brain activity induced by brushing stimulation, a block design of 29 min duration was used, including 14 stimulation blocks of $60 \mathrm{~s}$, separated by rest periods of the same duration. High-resolution anatomical images were also acquired at the end of the experiment using a fast spin-echo sequence $(\mathrm{TR}=2.5 \mathrm{~s} ; \mathrm{TE}=13 \mathrm{~ms}$; $\mathrm{FOV}=3 \times 3 \mathrm{~cm}$; matrix $=256 \times 128$; number of slices $=5$; slice thickness $=0.7 \mathrm{~mm}$ ).

Images were analyzed using SPM 8 (Wellcome Trust Centre for Neuroimaging, London, UK; http://www.fil.ion. ucl.ac.uk/spm/). Preprocessing included realignment for movement correction and co-registration of functional images with anatomical images. Functional images were temporally filtered using a high-pass filter with a cutoff period of $128 \mathrm{~s}$. Based on our hypothesis, a region of interest (ROI) analysis was performed on the BOLD signal in the left and right parietal cortex and left and right NBM. ROIs (circles of $1-\mathrm{mm}$ in diameter) were placed in the parietal cortex (1.4 mm posterior, $2.5 \mathrm{~mm}$ lateral and $1.5 \mathrm{~mm}$ ventral to bregma) [31] and in the NBM (1.4 mm posterior, $3 \mathrm{~mm}$ lateral and $7.6 \mathrm{~mm}$ ventral to bregma). The location of the ROIs was determined based on a previous study that examined the effect of somatosensory stimulation applied to the hindlimb [29]. The ROI locations appear in Fig. 2.

\section{Stimulation of the skin}

Innocuous stimulation using a soft-hair brush was applied to the posterior aspect of the left hindlimb covering the area from the buttock to the sole of the paw. Brushing was performed manually and was paced with an auditory cue. In our previous study [21], repetitive electrical stimulation of the NBM for 100 min produced an increase in extracellular NGF levels. Therefore, $1 \mathrm{~Hz}$ stimulation for 100 min was chosen for the NGF experiments. On the other hand, results of our blood flow study [29] suggested that $1 \mathrm{~Hz}$ brushing activates NBM with a longer latency ( $>2 \mathrm{~min}$ ) than that of $3 \mathrm{~Hz}$ brushing $(<10 \mathrm{~s})$. Therefore, $3 \mathrm{~Hz}$ stimulation for $1 \mathrm{~min}$ was chosen for the fMRI experiments to repeat short-lasting stimulation for averaging and minimize the length of the experimental session. In the fMRI experiment, the animals' lower thighs were fixed to the holder with adhesive tapes, with stimulation applied mainly to the paw.
Drugs

In 7 NGF experiments, the nAChR antagonist mecamylamine (mecamylamine hydrochloride; Sigma, USA), which is blood-brain barrier (BBB) permeable, was used. Mecamylamine was dissolved in saline at a concentration of $20 \mathrm{mg} / \mathrm{ml}$, and administered intravenously through a femoral vein as a single dose at $100 \mathrm{~min}$ before the onset of the brushing stimulation. Ficoll 70 (Pharmacia Biotech, Sweden) was injected (i.v.) to compensate hypotension following mecamylamine injection. In preliminary experiments, we confirmed that mecamylamine did not produce systematic changes in NGF levels in cortical perfusate throughout $600 \mathrm{~min}$ after injection.

Statistical analysis

For statistical analysis, Prism 6 software (GraphPad Software, La Jolla, CA, USA) was used. Values are shown as mean \pm SE. The changes in NGF and BOLD (separately for each ROI) evoked by brushing were assessed by a paired $t$ test. The statistical significance level was set at $p<0.05$.

\section{Results}

Changes in extracellular NGF levels in the cerebral cortex in response to brushing

Extracellular NGF levels in the parietal cortex were measured in a total of 6 rats in the control condition without cholinergic blocker. Five rats were anesthetized with halothane and 1 rat was anesthetized with urethane. No differences were found across the two different anesthetics on basal levels and brushing-induced changes in NGF. Therefore, all data were pooled across different anesthetics. In all rats examined, the NGF concentration did not change during brushing, but increased gradually after the end of the stimulation. The NGF concentration was maximum in samples taken at 200-400 min after the cessation of brushing. The NGF concentration before stimulation was $14.9 \pm 4.0 \mathrm{pg} /$ $\mathrm{ml}$, and was increased significantly $(p<0.05)$ to $22.3 \pm 4.6 \mathrm{pg} / \mathrm{ml}$ in samples taken at 300-400 min after the stimulation ended (Fig. 1a).

Effects of a nAChR antagonist on the brushing-induced increase in extracellular NGF levels in the cerebral cortex

To define the role of cholinergic synapses in mediating brushing-induced increases in extracellular NGF, we examined whether a specific cholinergic receptor antagonist 


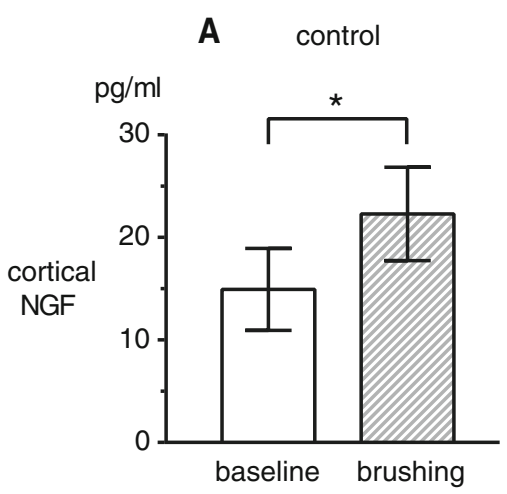

B mecamylamine

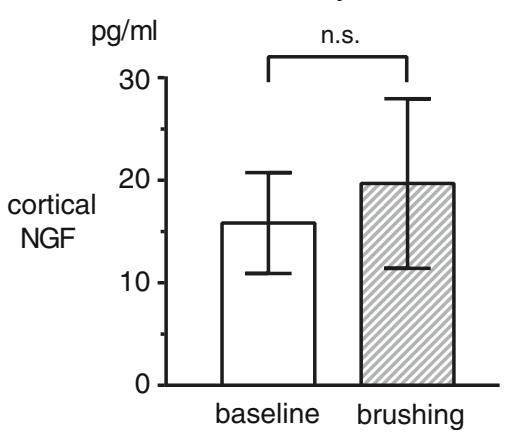

Fig. 1 Changes in NGF concentrations in cortical perfusate in the right parietal cortex induced by brushing of the left hindlimb. NGF concentrations in cortical perfusate taken before (white column) and after (hatched column) brushing. Each column and error bar shows the mean \pm SE. a Control rats without drugs $(n=6)$. b Rats pretreated with mecamylamine $(n=7)$. $* p<0.05$; significant difference between the values before (baseline) and after brushing

could reduce cortical responses to brushing. Mecamylamine, a nAChR antagonist, was used in 7 rats at $20 \mathrm{mg} / \mathrm{kg}$ (i.v.), which blocked the increase in NGF levels induced by NBM stimulation in our previous study [20]. Basal levels of extracellular NGF were $15.8 \pm 4.9 \mathrm{pg} / \mathrm{ml}$, which was similar to levels in control rats without the cholinergic receptor antagonist. However, the increase in extracellular NGF levels after brushing seen in control rats was not induced. The NGF concentration was $19.7 \pm 8.3 \mathrm{pg} / \mathrm{ml}$ in samples taken at 300-400 min after the stimulation ended (Fig. 1b). The NGF levels did not show any significant change after brushing compared to pre-stimulus levels $(p=0.7)$.

BOLD signal changes in the NBM in response to brushing

The location of the measured ROIs is shown in the inset diagram of the rat brain in Fig. 2. BOLD signal changes in the parietal cortices and NBMs induced by 1-min nonnoxious brushing at a frequency of $3 \mathrm{~Hz}$ were averaged in 5 rats. Non-noxious brushing was applied to the left hindlimb. During brushing, cortical BOLD signals increased in the right hemisphere on the contralateral side of the stimulated hindlimb (Fig. 2b). Changes in cortical BOLD signals occurred within $10 \mathrm{~s}$ from the onset of brushing and remained increased until the end of the stimulation. In contrast, BOLD signal in the ipsilateral parietal cortex did not increase (Fig. 2a).

In the NBM contralateral to the stimulated hindlimb, the BOLD signal was increased during brushing (Fig. 2d). BOLD signals increase in the contralateral NBM was observed during the first $5 \mathrm{~s}$ and it remained increased throughout the stimulation period. The averaged BOLD signal during the whole brushing period was significantly higher than the prestimulus control signal $(p<0.05)$ (Fig. 3). The BOLD signal tended to increase in the ipsilateral NBM, but this effect did not reach statistical significance ( $p=0.29$ ) (Fig. 3). To further determine whether the NBM response was lateralized between left (ipsilateral side of stimulated hindlimb) and right (contralateral) hemispheres, the BOLD signal in the NBM of each hemisphere during brushing was compared (Fig. 3). Changes in the BOLD signal in the contralateral NBM were consistently and significantly larger compared to those of the ipsilateral NBM $(p<0.05)$.

\section{Discussion}

Our results show that NGF secretion in the parietal cortex could be evoked by somatosensory stimulation. Our findings that (1) NGF increases are first detectable hours after brushing, and (2) the NGF response was reduced by a nAChR blocker are consistent with the NGF response induced by electrical stimulation of the NBM [20, 21]. Therefore, it is most likely that cholinergic inputs from the NBM contribute to the secretion of NGF by somatosensory stimulation. Accordingly, we also showed that hindlimb stimulation increased BOLD signal in the NBM.

Secreted NGF is essential for cholinergic neurons to maintain their properties [32, 33]. For example, i.c.v. injection of NGF reverses cholinergic neuron atrophy and improves the learning abilities of aged rats with impaired spatial memory [16]. In addition, cortical injection of NGF antibodies impairs acetylcholine release and disrupts the learning abilities in adult rats [18]. These studies together with our studies $[20,21]$ indicate that there is a bidirectional relationship between basal forebrain cholinergic neurons and the neurons in cortical areas where they project, through NGF. NGF prevents glucose deprivationinduced or glutamate-induced neuronal damage in cortical cell cultures $[17,34]$ at concentrations well within the estimated concentrations in the cortical extracellular fluid [20]. Therefore, the secretion of NGF induced by somatic 


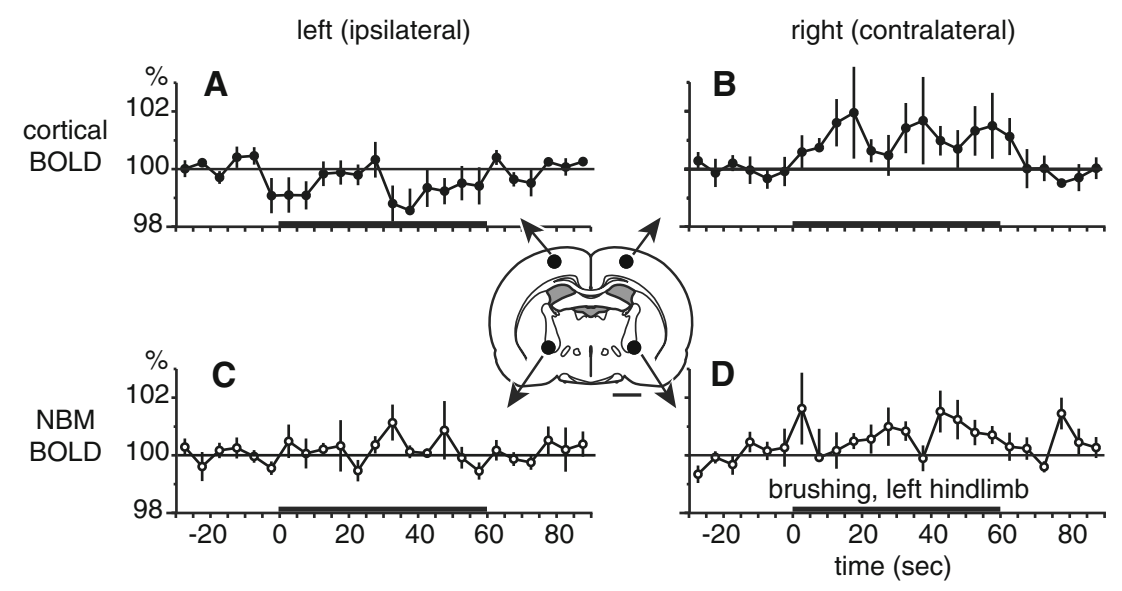

Fig. 2 Changes in BOLD signals in the parietal cortex and the NBM induced by brushing of the left hindlimb. BOLD signals during the 2-min trial measured every $5 \mathrm{~s}$ in the parietal cortex $(\mathbf{a}, \mathbf{b})$ and the $\operatorname{NBM}(\mathbf{c}, \mathbf{d})$, ipsilateral $(\mathbf{a}, \mathbf{c})$ and contralateral $(\mathbf{b}, \mathbf{d})$ to the stimulated hindlimb, expressed as a percentage of prestimulus control signal

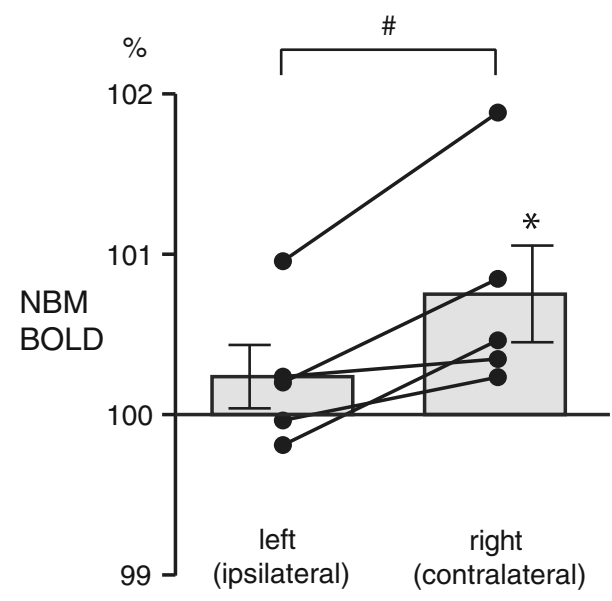

Fig. 3 Changes in BOLD signals in the NBM during brushing of the left hindlimb. Mean values of BOLD during brushing are plotted as the percentage of prestimulus control value (averages of $30 \mathrm{~s}$ before stimulation). Each column and error bar shows the mean $\pm \mathrm{SE}$ $(n=5)$. Each point indicates individual data. Each data obtained simultaneously is connected by a line. $* p<0.05$; significantly different compared to the prestimulus control value (statistical analysis was performed using absolute values). ${ }^{\#} p<0.05$; significantly different between values

sensory stimulation as shown in this study may be useful for maintaining the functions of cortical neurons and NBM cholinergic neurons essential for cognitive functions. The results of this study may explain why repeated somatosensory stimulation improves cerebral function in demented or post-stroke conditions [8-15]. Furthermore, non-noxious stimulation of the skin in daily life may contribute to maintain cognitive function by increasing NGF. $(n=5)$. Averages of $30 \mathrm{~s}$ before the brushing are used as prestimulus control signal. The thick horizontal lines on the abscissa indicate the 1-min period of stimulation. BOLD signal was extracted from each ROI illustrated by black circles in the inset of a coronal slice at $1.4 \mathrm{~mm}$ posterior to bregma. Scale bar below inset $2 \mathrm{~mm}$

Non-noxious cutaneous stimulation may promote the secretion of various hormones and neurotransmitters, such as CRH [35], oxytocin [36], ACh [37], and dopamine [38], from neurons in the brain. However, our results are the first evidence indicating that somatosensory stimulation promotes the secretion of neurotrophic factors. Time delay between BOLD changes and NGF increase (this study) are similar to delay between electrical stimulation of the NBM and NGF increase [20], and can be explained by synthesis of NGF proteins via mRNA. Neeper et al. [39] showed that NGF mRNA levels increased in the hippocampus and caudal neocortex following 2-7 nights with running wheels. Thus, brushing of the hindlimb may also increase expression of the NGF in the parietal cortex.

In the present study, somatosensory-evoked BOLD signal changes were investigated by functional MRI. Innocuous brushing of the hindlimb induced an increase in the BOLD signal of the contralateral parietal cortex over the representation of the hindlimb, as expected from previous studies [2226], and also of the contralateral NBM. This is the first study to show that somatosensory stimulus evoked BOLD signal changes in the NBM. The latency of the BOLD response in NBM was earlier than that in the parietal cortex. This result is consistent with the finding that blood flow increase in the parietal cortex induced by electrical stimulation of the NBM starts a few seconds after the onset of stimulation [40, 41]. Since an increase in BOLD signal usually correlates with an increase in neuronal activities [42, 43], the present result supports the above assumption that NBM neurons are activated by non-noxious cutaneous stimulation.

Changes in BOLD signal were significantly larger in the NBM in the contralateral compared with the ipsilateral side 
of the stimulated hindlimb. This result indicates that nonnoxious skin stimulation activates the contralateral NBM predominantly. Accordingly, hindlimb brushing increases NGF secretion (this study) and ACh release measured on the contralateral parietal cortex [37]. Furthermore, a brushing-induced increase in regional blood flow evoked in the contralateral parietal cortex, over the representation of the stimulated hindlimb, was decreased in the hemisphere ipsilateral to muscimol inactivation of NBM [29]. In addition, histological studies have shown that fibers from the NBM project unilaterally to ipsilateral cerebral cortices [44, 45]. Furthermore, although NBM innervation of the somatosensory cortex is mostly cholinergic in the rat, and each cortical area receives cholinergic afferents from neurons widely distributed within the NBM, each NBM neuron projects to a restricted cortical area without significant collateralization to adjacent subdivisions [46]. However, ascending neural pathways from cutaneous mechanoreceptors to the contralateral NBM are unknown. Future studies are needed to clarify the specific neural pathway from the limbs to the contralateral NBM.

\section{Limitations}

One limitation of this study is that the difference of stimulus frequency between the NGF studies $(1 \mathrm{~Hz}$ for $100 \mathrm{~min}$ ) and the fMRI studies ( $3 \mathrm{~Hz}$ for $1 \mathrm{~min}$ ) may make it harder to link the BOLD signal results with the NGF data. However, in our previous experiments, brushing at $1 \mathrm{~Hz}$ continued for 15 min produced an increase of cortical blood flow equivalent to (or even larger than) that produced by brushing at $3 \mathrm{~Hz}$ for $3 \mathrm{~min}$ [29]. Therefore, it can be assumed that brushing at $1 \mathrm{~Hz}$ for $100 \mathrm{~min}$ would produce increases in BOLD signals equivalent to (or even larger than) that produced by brushing at $3 \mathrm{~Hz}$ for $1 \mathrm{~min}$.

Another limitation is that we did not test the effect of a nAChR antagonist that does not permeate the blood-brain barrier, so that we cannot exclude the possibility that blocking transmission at autonomic ganglia that may influence sympathetic and parasympathetic tone are involved in the present NGF response. However, this possibility is less likely because autonomic nerve fibers innervate blood vessels on the cortical surface, but do not reach the cortical parenchyma. In addition, we did not examine the effect of mecamylamine on BOLD signal changes during brushing. However, we observed (data not shown) that brushing-induced increases in parietal cortical blood flow persisted after i.v. injection of mecamylamine (but were reduced by atropine). Therefore, the effect of mecamylamine on brushing-induced increases in NGF does not appear to be due to abolishing blood flow changes or transmission of somatosensory input from the

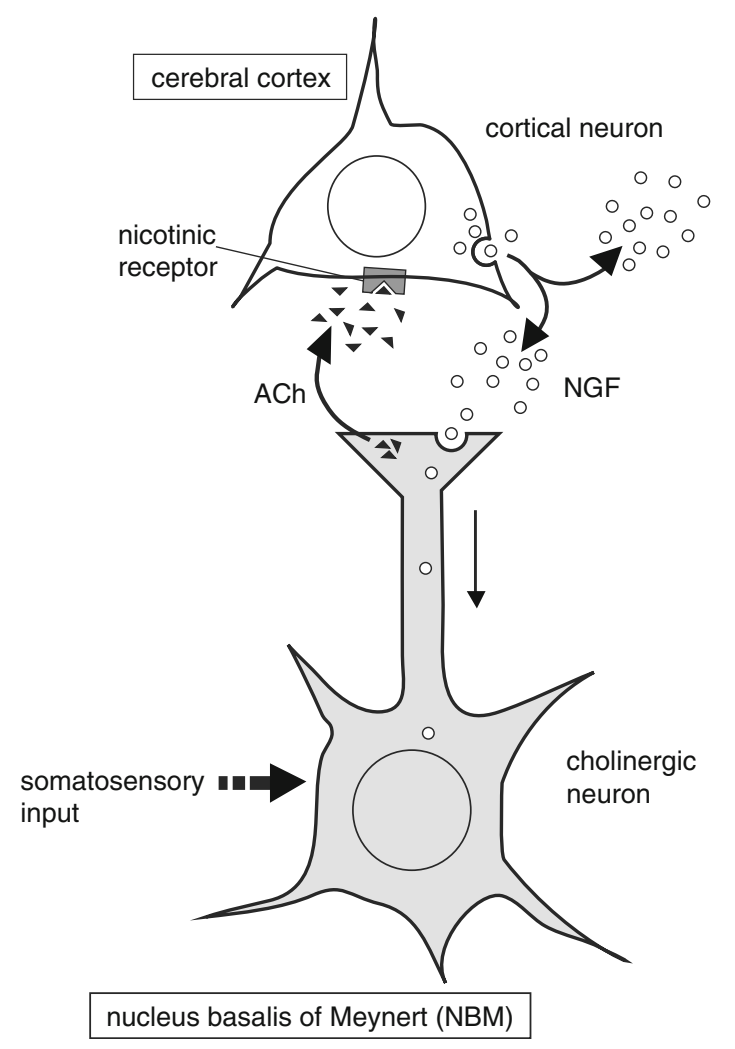

Fig. 4 Schematic diagram showing the proposed mechanisms by which NGF secretion occurs in the cerebral cortex in response to nonnoxious somatosensory stimulation. Non-noxious somatosensory input activates the NBM, and cholinergic inputs from there to the cortex promote NGF secretion from cortical neurons by activation of the nAChRs in the cerebral cortex

hindlimb, but most likely to blocking nAChRs receiving $\mathrm{ACh}$ released from cholinergic fibers originating in the NBM.

\section{Conclusion}

We propose that non-noxious mechanical stimulation applied to the skin activates the cholinergic system of the basal forebrain and promotes NGF secretion in the parietal cortex (Fig. 4). Secreted NGF may act to maintain neuronal function at the appropriate levels. Although direct evidence of neuronal excitation by means of electrophysiological technique is lacking, the present results on cortical NGF responses and BOLD changes in the NBM are consistent with and strongly suggest that non-noxious cutaneous stimulation activates the NBM neurons. The present animal fMRI study results may be advantageous for the translation towards human fMRI studies for screening appropriate stimulation that activates the NBM, leading to the maintenance of normal cognitive ability. 
Acknowledgment This work was supported in part by a grant from the Smoking Research Foundation.

Conflict of interest The authors declare that they have no conflict of interest.

Open Access This article is distributed under the terms of the Creative Commons Attribution License which permits any use, distribution, and reproduction in any medium, provided the original author(s) and the source are credited.

\section{References}

1. Hotta H, Uchida S, Shimura M, Suzuki H (1999) Uterine contractility and blood flow are reflexively regulated by cutaneous afferent stimulation in anesthetized rats. J Auton Nerv Syst 75:23-31

2. Hotta H, Schmidt RF, Uchida S, Watanabe N (2010) Gentle mechanical skin stimulation inhibits the somatocardiac sympathetic C-reflex elicited by excitation of unmyelinated C-afferent fibers. Eur J Pain 14:806-813

3. Hotta H, Masunaga K, Miyazaki S, Watanabe N, Kasuya Y (2012) A gentle mechanical skin stimulation technique for inhibition of micturition contractions of the urinary bladder. Auton Neurosci 167:12-20

4. Hotta H (2013) Neural mechanisms of inhibition of vesical micturition contractions by gentle cutaneous stimulation. J Physiol Sci 63(suppl 1):S63

5. Sato A, Sato Y, Schmidt RF (1997) The impact of somatosensory input on autonomic functions. Rev Physiol Biochem Pharmacol 130:1-328

6. Watanabe N, Miyazaki S, Mukaino Y, Hotta H (2012) Effect of gentle cutaneous stimulation on heat-induced autonomic response and subjective pain intensity in healthy humans. J Physiol Sci $62: 343-350$

7. Watanabe N (2013) Effects of gentle skin stimulation on somatocardiovascular reflexes and contribution of emotions. J Physiol Sci 63(suppl 1):S13

8. Scherder E, Bouma A, Steen L (1995) The effects of peripheral tactile stimulation on memory in patients with probable Alzheimer's disease. Am J Alzheimer's Dis 10:15-21

9. Guo Y, Shi X, Uchiyama H, Hasegawa A, Nakagawa Y, Tanaka M, Fukumoto I (2002) A study on the rehabilitation of cognitive function and short-term memory in patients with Alzheimer's disease using transcutaneous electrical nerve stimulation. Front Med Biol Eng 11:237-247

10. Cooper EB, Scherder EJA, Cooper JB (2005) Electrical treatment of reduced consciousness: experience with coma and Alzheimer's disease. Neuropsychol Rehabil 15:389-405

11. Hummelsheim H, Hauptmann B, Neumann S (1995) Influence of physiotherapeutic facilitation techniques on motor evoked potentials in centrally paretic hand extensor muscles. Electroencephalogr Clin Neurophysiol 97:18-28

12. Hunter SM, Crome P, Sim J, Pomeroy VM (2008) Effects of mobilization and tactile stimulation on recovery of the hemiplegic upper limb: a series of replicated single-system studies. Arch Phys Med Rehabil 89:2003-2010

13. Gibb RL, Gonzalez CLR, Wegenast W, Kolb BE (2010) Tactile stimulation promotes motor recovery following cortical injury in adult rats. Behav Brain Res 214:102-107

14. Kawahira K, Shimodozono M, Etoh S, Kamada K, Noma T, Tanaka N (2010) Effects of intensive repetition of a new facilitation technique on motor functional recovery of the hemiplegic upper limb and hand. Brain Inj 24:1202-1213
15. Yamauchi T, Shiraki M, Urakawa S, Jung C-G, Hida H, Nishijo H, Ono T, Nishino H (2013) Continuous grasping of polyethylene-fiber cushion induced prompt recovery of hand contracture and rigidity due to cerebral infarction. J Physiol Sci 63(suppl 1): S150

16. Fischer W, Wictorin K, Björklund A, Williams LR, Varon S, Gage FH (1987) Amelioration of cholinergic neuron atrophy and spatial memory impairment in aged rats by nerve growth factor. Nature 329:65-68

17. Cheng B, Mattson MP (1991) NGF and bFGF protect rat hippocampal and human cortical neurons against hypoglycemic damage by stabilizing calcium homeostasis. Neuron 7:1031-1041

18. Gutiérrez H, Miranda MI, Bermúdez-Rattoni F (1997) Learning impairment and cholinergic deafferentation after cortical nerve growth factor deprivation. J Neurosci 17:3796-3803

19. Lee Y-S, Danandeh A, Baratta J, Lin C-Y, Yu J, Robertson RT (2013) Neurotrophic factors rescue basal forebrain cholinergic neurons and improve performance on a spatial learning test. Exp Neurol 249:178-186

20. Hotta H, Kagitani F, Kondo M, Uchida S (2009) Basal forebrain stimulation induces NGF secretion in ipsilateral parietal cortex via nicotinic receptor activation in adult, but not aged rats. Neurosci Res 63:122-128

21. Hotta H, Uchida S, Kagitani F (2007) Stimulation of the nucleus basalis of Meynert produces an increase in the extracellular release of nerve growth factor in the rat cerebral cortex. J Physiol Sci 57:383-387

22. Boakye M, Huckins SC, Szeverenyi NM, Taskey BI, Hodge CJ Jr (2000) Functional magnetic resonance imaging of somatosensory cortex activity produced by electrical stimulation of the median nerve or tactile stimulation of the index finger. J Neurosurg 93:774-783

23. Chang C, Shyu B-C (2001) A fMRI study of brain activations during non-noxious and noxious electrical stimulation of the sciatic nerve of rats. Brain Res 897:71-81

24. Sachdev RNS, Champney GC, Lee H, Price RR, Pickens DR 3rd, Morgan VL, Stefansic JD, Melzer P, Ebner FF (2003) Experimental model for functional magnetic resonance imaging of somatic sensory cortex in the unanesthetized rat. Neuroimage 19:742-750

25. Hlushchuk Y, Hari R (2006) Transient suppression of ipsilateral primary somatosensory cortex during tactile finger stimulation. J Neurosci 26:5819-5824

26. Lowe AS, Beech JS, Williams SCR (2007) Small animal, whole brain fMRI: innocuous and nociceptive forepaw stimulation. Neuroimage 35:719-728

27. Mouraux A, Diukova A, Lee MC, Wise RG, Iannetti GD (2011) A multisensory investigation of the functional significance of the "pain matrix". Neuroimage 54:2237-2249

28. Hotta H, Watanabe N, Piché M, Hara S, Uchida S, Yokawa T (2014) Non-noxious skin stimulation activates the nucleus basalis of Meynert and promotes NGF secretion in the parietal cortex via nicotinic ACh receptors in anesthetized rats. J Physiol Sci 64(suppl 1):S243

29. Piché M, Uchida S, Hara S, Aikawa Y, Hotta H (2010) Modulation of somatosensory-evoked cortical blood flow changes by GABAergic inhibition of the nucleus basalis of Meynert in urethane-anaesthetized rats. J Physiol 588:2163-2171

30. Marksteiner J, Pirchl M, Ullrich C, Oberbauer H, Blasko I, Lederer W, Hinterhuber H, Humpel C (2008) Analysis of cerebrospinal fluid of Alzheimer patients. Biomarkers and toxic properties. Pharmacology 82:214-220

31. Paxinos GE, Watson $C$ (2007) The rat brain in stereotaxic coordinates, 6th edn. Elsevier, Amsterdam

32. Debeir T, Saragovi HU, Cuello AC (1999) A nerve growth factor mimetic TrkA antagonist causes withdrawal of cortical 
cholinergic boutons in the adult rat. Proc Natl Acad Sci USA 96:4067-4072

33. Cuello AC, Bruno MA, Allard S, Leon W, Iulita MF (2010) Cholinergic involvement in Alzheimer's disease. A link with NGF maturation and degradation. J Mol Neurosci 40:230-235

34. Shimohama S, Ogawa N, Tamura Y, Akaike A, Tsukahara T, Iwata H, Kimura J (1993) Protective effect of nerve growth factor against glutamate-induced neurotoxicity in cultured cortical neurons. Brain Res 632:296-302

35. Hotta H, Sato A, Sumitomo T (1992) Hypothalamic corticotropin-releasing hormone $(\mathrm{CRH})$ secretion into hypophysial portal blood is regulated by cutaneous sensory stimulation in anesthetized rats. Jpn J Physiol 42:515-524

36. Stock S, Uvnäs-Moberg K (1988) Increased plasma levels of oxytocin in response to afferent electrical stimulation of the sciatic and vagal nerves and in response to touch and pinch in anaesthetized rats. Acta Physiol Scand 132:29-34

37. Kurosawa M, Sato A, Sato Y (1992) Cutaneous mechanical sensory stimulation increases extracellular acetylcholine release in cerebral cortex in anesthetized rats. Neurochem Int 21:423-427

38. Maruyama K, Shimoju R, Ohkubo M, Maruyama H, Kurosawa M (2012) Tactile skin stimulation increases dopamine release in the nucleus accumbens in rats. J Physiol Sci 62:259-266

39. Neeper SA, Gómez-Pinilla F, Choi J, Cotman CW (1996) Physical activity increases mRNA for brain-derived neurotrophic factor and nerve growth factor in rat brain. Brain Res 726:49-56
40. Biesold D, Inanami O, Sato A, Sato Y (1989) Stimulation of the nucleus basalis of Meynert increases cerebral cortical blood flow in rats. Neurosci Lett 98:39-44

41. Hotta H, Uchida S, Kagitani F, Maruyama N (2011) Control of cerebral cortical blood flow by stimulation of basal forebrain cholinergic areas in mice. J Physiol Sci 61:201-209

42. Logothetis NK, Pauls J, Augath M, Trinath T, Oeltermann A (2001) Neurophysiological investigation of the basis of the fMRI signal. Nature 412:150-157

43. Lee JH, Durand R, Gradinaru V, Zhang F, Goshen I, Kim D-S, Fenno LE, Ramakrishnan C, Deisseroth K (2010) Global and local fMRI signals driven by neurons defined optogenetically by type and wiring. Nature 465:788-792

44. Lehmann J, Nagy JI, Atmadja S, Fibiger HC (1980) The nucleus basalis magnocellularis: the origin of a cholinergic projection to the neocortex of the rat. Neuroscience 5:1161-1174

45. Luiten PGM, Gaykema RPA, Traber J, Spencer DG Jr (1987) Cortical projection patterns of magnocellular basal nucleus subdivisions as revealed by anterogradely transported Phaseolus vulgaris leucoagglutinin. Brain Res 413:229-250

46. Baskerville KA, Chang HT, Herron P (1993) Topography of cholinergic afferents from the nucleus basalis of Meynert to representational areas of sensorimotor cortices in the rat. J Comp Neurol 335:552-562 